\title{
Children in clinical trials: towards evidence- based pediatric pharmacotherapy using pharmacokinetic-pharmacodynamic modeling
}

Janneke M Brussee, Elisa AM Calvier, Elke HJ Krekels, Pyry AJ Välitalo, Dick Tibboel, Karel Allegaert \& Catherijne AJ Knibbe

To cite this article: Janneke M Brussee, Elisa AM Calvier, Elke HJ Krekels, Pyry AJ Välitalo, Dick Tibboel, Karel Allegaert \& Catherijne AJ Knibbe (2016): Children in clinical trials: towards evidence-based pediatric pharmacotherapy using pharmacokinetic-pharmacodynamic modeling, Expert Review of Clinical Pharmacology

To link to this article: http://dx.doi.org/10.1080/17512433.2016.1198256

Accepted author version posted online: 08 Jun 2016.

Published online: 08 Jun 2016.

Submit your article to this journal $₫$

Q View related articles $\sqsubset$

View Crossmark data 
Publisher: Taylor \& Francis

Journal: Expert Review of Clinical Pharmacology

DOI: $10.1080 / 17512433.2016 .1198256$

\section{Review}

\section{Children in clinical trials: towards evidence-based pediatric pharmacotherapy using pharmacokinetic-pharmacodynamic modeling}

Janneke M Brussee ${ }^{1}$, Elisa AM Calvier ${ }^{1}$, Elke HJ Krekels ${ }^{1}$, Pyry AJ Välitalo ${ }^{1}$, Dick Tibboel ${ }^{2}$, Karel Allegaert $^{2,3}$, Catherijne AJ Knibbe ${ }^{1,4}$

1 Division of Pharmacology, Leiden Academic Centre for Drug Research, Leiden University, Leiden, The Netherlands

2 Intensive Care and Department of Surgery, Erasmus MC-Sophia Children's Hospital, Rotterdam, the Netherlands

3 Department of Development and Regeneration, KU Leuven, Belgium

4 Department of Clinical Pharmacy, St. Antonius Hospital, Nieuwegein, The Netherlands

Contact details authors:

brusseejm@lacdr.leidenuniv.nl

e.a.m.calvier@lacdr.leidenuniv.nl

e.krekels@lacdr.leidenuniv.nl

p.a.j.valitalo@lacdr.leidenuniv.nl

d.tibboel@erasmusmc.nl

karel.allegaert@uzleuven.be

c.knibbe@antoniusziekenhuis.nl (corresponding author)

\section{Corresponding author:}

C.A.J. Knibbe

Department of Clinical Pharmacy, St. Antonius Hospital, Nieuwegein, The Netherlands

c.knibbe@antoniusziekenhuis.nl 


\section{Abstract}

Introduction: In pediatric pharmacotherapy, many drugs are still used off-label, and their efficacy and safety is not well characterized. Different efficacy and safety profiles in children of varying ages may be anticipated, due to developmental changes occurring across pediatric life.

Areas covered: Beside pharmacokinetic (PK) studies, pharmacodynamic (PD) studies are urgently needed. Validated PKPD models can be used to derive optimal dosing regimens for children of different ages, which can be evaluated in a prospective study before implementation in clinical practice. Strategies should be developed to ensure that formularies update their drug dosing guidelines regularly according to the most recent advances in research, allowing for clinicians to integrate these guidelines in daily practice.

Expert Commentary: We anticipate a trend towards a systems-level approach in pediatric modeling to optimally use the information gained in pediatric trials. For this approach, properly designed clinical PKPD studies will remain the backbone of pediatric research.

\section{Keywords}

Pediatrics, clinical trial, off-label, maturation, PKPD, physiology, evidence-based dose recommendations, dose individualization, pharmacotherapy 


\section{Pharmacotherapy in clinical practice}

A major problem in pediatric healthcare is that drugs are largely used in an unlicensed or off-label manner (1). For many drugs, the pharmacokinetic, efficacy and safety profile in children is not very well characterized (2), resulting in a much lower level of evidence of drug efficacy and safety in pediatric pharmacotherapy compared to adult pharmacotherapy. This review focuses more on current drugs used in clinical practice, rather than how to bring new drugs to the pediatric market.

In children, fewer and smaller studies on drug pharmacology are performed than in adults. This can be attributed to the ethical and practical limitations of pediatric trials $(3,4)$. As it is considered to be unethical to study drugs in healthy children, all pharmacological research in children involves diseased children of which the potential number of participants is limited while age may vary largely. Next, informed consent from the parents or legal guardians is needed and if children are old enough to understand, also informed assent is needed from the patients after informing them about the trial in such a manner they understand. Practical limitations of a trial include the limited number and volume of plasma samples that can be obtained per patient (e.g. the accepted rule that not more than $3 \%$ of circulating volume can be harvested during the course of the study), as well as the challenges related to obtain specimen at the extremes of childhood (e.g. extreme low birthweight infants). Furthermore, it is hard to find validated endpoints to measure efficacy in the pediatric population (5). Finally, both the dose that is used and sampling times are often limited to the patients' need in clinical care, which may not be optimal from a research point of view.

In absence of results of dedicated pediatric studies, pediatric doses have been scaled from adult pharmacokinetic (PK) and pharmacodynamic (PD) data using different methods $(6,7)$. These methods include empirical approaches (e.g. linear or allometric extrapolation on the basis of body weight or body surface area), or mechanistic modeling in which physiologically based pharmacokinetic (PBPK) models $(8,9)$ are used. Maturation functions that have been derived across the neonatal or pediatric age range, for instance for GFR in neonates(10) or for clearance in the youngest patients(11) , are largely data-driven and empirical. Mechanistic scaling methods have distinct advantages over empirical methods (12), however to date no single mechanistic method has been found suitable across the entire pediatric age range (13). Empirically determined dosages are typically expressed in $\mathrm{mg} / \mathrm{kg}$ and have usually been adjusted based on clinical experience after which they are summarized in national formularies. It should be realized however, that this approach may not yield a dose with an optimal efficacy/safety profile, and that both during and after the process of optimizing a dose, the patients are at risk for over- and underdosing. 


\section{Developmental changes influencing drug pharmacology}

Inter-individual variability in pharmacokinetics (PK) and pharmacodynamics (PD) in the pediatric population is typically due to age and size, but can also for instance result from pharmacogenetic differences, disease or drug-drug interactions. Because developmental changes affect the pharmacology of administered compounds $(14,15)$, children who can vary in age between 0 and 18 years of age are an even more heterogeneous population than adults. As such, in children altered PK and/or PD and toxicity due to exaggerated PD effects or off-target effects may be expected (16). This is especially relevant in the youngest age groups such as (prematurely born) neonates, as this is where the changes are the largest $(17,18)$.

These developmental changes include maturation of enzyme expression activity influencing metabolic clearance of drugs, and age-related changes in glomerular filtration rate (GFR) and active tubular processes influencing renal excretion (19). Metabolizing enzymes such as P450 cytochromes (CYP) and UDP-glucuronosyltranferases (UGTs) may have different maturation profiles, as enzyme abundance increases or for some enzymes decreases with age $(20,21)$. Furthermore, depending on the drug properties, drug metabolism is also affected by hepatic blood flow and/or the unbound drug fraction (22), which also change throughout childhood. GFR is mostly influenced by renal blood flow (23) as has been described by many empirical formulas and models (24). Total renal clearance is also affected by passive and active transporters in the tubular epithelium. Moreover, the drug-protein binding to albumin and $\alpha 1$-acid glycoprotein increases with increasing age, due to an increase in protein expression and binding capacity. This results in an decreased unbound drug fraction $(25,26)$, although for most drugs changes in protein binding have no clinical consequence.

In addition to the influence of age on elimination, age-related changes in body composition may also be of influence on the distribution of drugs. The body water to fat ratio is higher in neonates and young infants, as they have a larger amount of extracellular fluid and total-body water compared to adults (17). The exact implications of these changes for drug PK profiles are dependent on drug

\section{properties.}

For drugs that are taken orally, the absorption process may also be subject to changes. The absorption rate of most drugs is slower in neonates and young infants than in older children, due to delayed gastric emptying and lower intestinal motility (17). Especially in early life, gastric emptying is delayed compared to older children and adults, potentially delaying absorption in this population as well (27). Also, the gastric $\mathrm{pH}$ in neonates may be increased after oral feeding (28), which may affect the bioavailability of some drugs $(15,17)$. Furthermore, it is assumed that intestinal surface area and 
age-associated blood flow in the intestinal mucosa is reduced in young infants (17). Unfortunately, developmental changes affecting drug absorption in infants and children have not been well-studied (17).

Drug response can be increased or decreased during maturation and growth of a child due to developmental changes in expression and function of receptors and post-receptor mechanisms involved in drug action, or population specific pathophysiology. This requires dedicated studies to quantify the effects of these changes. Additionally, risk for drug toxicity in children can be increased or decreased due to PK and/or PD ontogeny and the efficacy-toxicity trade-off could be different in children (29).

\section{Developing evidence-based dosing regimens for children}

Due to anticipated drug dependent PK and PD changes among children of different ages and the current lack of knowledge on organ specific ontogeny processes (30), optimal drug efficacy and safety cannot be guaranteed when pediatric drug doses are derived from adult data (31). Instead, pediatric PKPD models should be developed in order to propose dosing regimens, across the pediatric population. In order to get to evidence-based dosing regimens for children, a multi-step approach (Figure 1) (32) has been proposed. This multi-step approach is based on the learningconfirming principle (33) and it relies on the population approach (32).

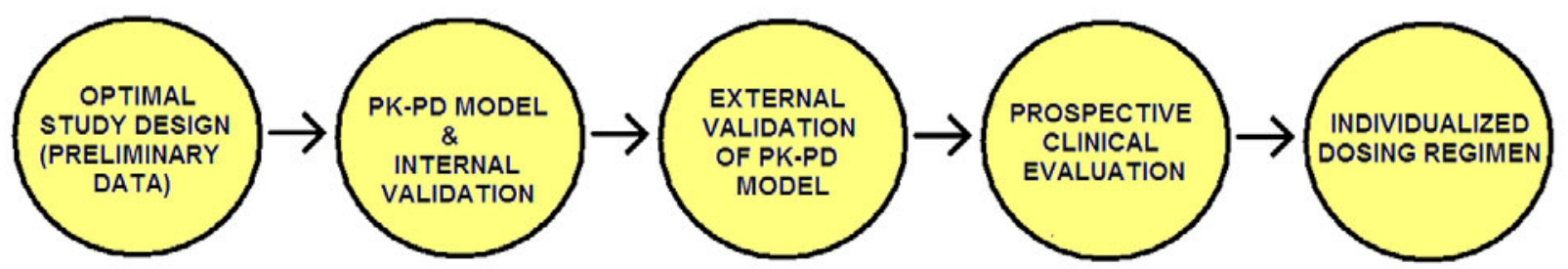

Figure 1. Proposed multistep approach for the optimization of drug dosing in children. The four steps that are proposed are [1] optimization of clinical trial designs based on simulations using preliminary data; [2] development and internal validation of population PKPD models using sparse data; [3] external validation of the population PKPD models using independent data; and [4] prospective clinical evaluation of the PKPD model-based dosing regimen. PK, pharmacokinetics; PD, pharmacodynamics. Printed with permission from Ince l et al. Drug Discovery Today 2009;14:316-20 (32).

Over the last decades, the population approach which is based on non-linear mixed effect modeling (34-36), has gained in popularity. Using this approach, models are developed to estimate population 
PK and/or PD parameters in an entire population of patients simultaneously, while still taking into account that different observations come from different patients. This approach allows for the characterization of general trends in the population and also for differentiation between interindividual variability and residual or intra-individual variability within a single model (37). The latter is an advantage over the standard two-stage approach, in which first the PK or PD parameters are fit to observed data in each individual separately, requiring dense sampling, after which these individual parameters are presented using summary statistics like mean and standard error. Moreover, the population approach with its more statistically powerful description of inter-individual variability allows for a covariate analysis in which patient characteristics that can explain (part of) the interindividual variability can be identified (38).

Particularly in children, the population approach as a tool in PK and/or PKPD analyses is preferred over the standard two-stage approach, because of limited sampling possibilities in individual children, known inter-individual variability that may or may not be related to age and advanced possibilities for so-called covariate analyses (39-41). This is particularly relevant for very young patients, such as term and preterm neonates, from whom only sparse samples can be collected. As a result, the population approach is the recommended approach by the EMA $(42,43)$ and FDA $(44)$ for the analysis of pediatric PKPD data and the development of evidence-based dosing regimens. Finally, this approach is found to be superior over the standard-two stage approach (40) and also allows, when deemed appropriate, for the use of adult data as priors to lower the sample size needed and/or increase the statistical power of the study (45).

The pharmacometric field develops mathematical models of physiology, pharmacology and/or disease to describe and quantify interactions between drugs and patients, including PK, PD and toxicity effects of both new and commonly used drugs. In pharmacometrics, most efforts have been focused on PK modeling, while in clinical care and drug development PK, PD and toxicity should be considered $(42,44,46)$. As stated above, the concentration-effect relationship may change with age because of increased or decreased expression and function of receptors involved in drug action and response. For new drugs, the FDA and EMA developed a decision tree for the evaluation of the need for (the type of) pediatric trials $(44,47,48)$. When it is reasonable to assume that children and adults have a similar disease progression and a similar response to intervention and it is also reasonable to assume similar concentration-response in both populations, then only PK studies to achieve plasma concentrations similar to adults and safety trials should be conducted. When only disease progression and response to intervention are assumed similar, PKPD studies to get concentrationresponse information are necessary in addition to the previously mentioned PK and safety studies. 
When disease progression and response to intervention are not assumed to be similar in pediatrics and adults, then both PK studies and safety/efficacy trials should be performed (48). For example, in infectious diseases, most model approaches are focused on development of a PK model to study PK maturation. These PK models are then used for simulations to give dose recommendations based on a target concentration pre-defined based on adult or in vitro data, rather than performing a PKPD trial (49).

For some drugs with a low PK variability and/or when it is not possible to collect all required PK information, the dose can be also directly related to the PD with K-PD models (50) which can be, with extra caution, then used to inform dosing. However, when PKPD models can be identified, they should be preferred over K-PD models (51). Below we describe the different steps of figure 1 in order to get to evidence-based dosing guidelines in children.

\section{Study design and data collection}

Just as in any other research field, a proper study design is pivotal in answering the research questions of pharmacological studies. The study design should be such that the parameters in the anticipated model are identifiable. Because practice and theory often do not match, the design of a study should be discussed within a multidisciplinary team including clinicians, pharmacists, nurses and pharmacometricians. Commonly, inter- and intra-individual variability in PKPD is higher in children and especially in neonates compared to adults, which is due to maturation aspects and inter-individual differences in weight (52). Moreover, patients numbers are typically lower and as a result both these aspects should be accounted for in the study design as it causes the statistical power of analysis to decrease.

\subsection{Structural jentifiability}

Structural identifiability is concerned with whether the parameters of a postulated model can be identified from a specified experiment or experiments with perfect input-output data (53). For instance, identifiability issues can arise when plasma metabolite concentrations are included in the model. Metabolite concentrations are mostly studied in special populations when the metabolite is suspected to be active or when a certain elimination pathway is being studied. Trial design can solve structural parameter identifiability issues by, for example, the use of different routes of administration or sampling of different media (e.g. blood and urine samples). For example, , without urine sampling upon a single dose over a long time interval to estimate the fraction of the dose that is metabolized through the involved pathway, identifiability issues in quantifying the metabolism and excretion pathways of the parent compound may occur. An example of this problem including how 
to address this issue has been described elsewhere (54). In some cases, structural identifiability issues cannot be addressed by any method, which requires proper discussion of the specific limitations before starting the trial (54).

\subsection{Numerical identifiability}

Numerical identifiability relates to the informativeness of the data and can be improved by the optimization of study design and its execution (55). The impact of the study design on the precision and bias of the estimated parameters of a population model in adults is well established (56-60). In order to minimize the parameter uncertainty, two main clinical trial optimization methods exist, namely clinical trial simulation and optimal design (61). Clinical trial simulation allows for the investigation of a limited number of candidate study designs by simulating datasets with a given design and fitting the specified model to those simulated datasets. The mean and variance of the parameter estimates are then calculated, to come up with the bias and precision, respectively, of the design. Optimal design methods involve the direct calculation of the expected parameter standard errors, which could be compared to performing a clinical trial simulation with an infinite number of simulated datasets. Several optimal design software exists and most of these have been evaluated by Nyberg et al. (62).

The optimization of all or specific PKPD parameters can be done over different clinical trial design parameters, like the number of samples, the number of patients included, the sampling times, the doses administered and patient characteristics $(63,64)$. The trial optimization can take into account practical and ethical (e.g. maximal number of samples per patients), as well as economic constraints (e.g. treatment costs). There is limited experience with all of these techniques in pediatric pharmacology research.

\subsubsection{Sampling}

Increased numbers of blood samples per patient will increase the accuracy and precision of the estimated model parameters and the estimated inter-individual and residual variability $(56,58,59)$. Depending on the practical constraints, specific sampling times or sampling windows can be optimized. A design allowing for variation in sampling time (design with multiple sampling windows) and dosages among subjects can be beneficial and protect against the ill-effects of model misspecification, as has been demonstrated before (57). Despite the use of clinical trial simulations, practical constraints might prevent the identifiability of some model parameters. For example, the impossibility to sample at early times would prevent the estimation of the absorption rate.

\subsubsection{Data collection}


When designing a PK(PD) study, information on patient characteristics (potential model covariates) that can correlate to PK or PD parameters need to be collected. Body weight and age are the most commonly used covariates in the pediatric population, to describe the influence of growth and development. For children younger than 2 years of age, gestational age, postmenstrual age, postconceptual age and postnatal age may be relevant covariates as well (65). Many other covariates which can influence the PK and /or PD of the drug studied, can be collected, such as disease state, comedications or the treatment with artificial devices such as extracorporeal membrane oxygenation (ECMO), ventricular assist devices or renal replacement $(66,67)$. In general, correlations between potential covariates should be avoided whenever possible, as the combination of two correlated covariates in a population model may result in bias in parameter estimates (68).

\subsubsection{Patient inclusion}

Several general design techniques allow to reduce bias, such as randomization and blinding. In general, potential confounders should be defined, such as co-treatment (e.g. inotropics in cardiac surgery patients), as they might bias the results of the analysis. The distribution of covariates likely to be included in the model should guide, whenever possible, patient inclusion in the trial (69) or trial arms, to prevent unbalanced designs hindering accuracy and precision (70). In a research protocol, it could be useful to identify strata to assure a spread in the covariate (e.g. age) values over the strata in order to gain information across the required range. When patients with only a limited range in covariate values are included, prediction to other patients outside this covariate range would require extrapolation. As interpolations are generally more reliable, the correlations of the covariates to PK or PD parameters can be extended to other subjects more reliably when the covariate values are spread and balanced.

\section{Population PKPD modeling and covariate analysis}

When the data are available for analysis, model development including a covariate analysis is performed to identify which factors can explain (part of) the observed inter-individual variability in the study. Possible covariates include demographic factors like age, body weight and gender or clinical factors like pharmacogenetics, disease state or comorbidities, or co-medication. In a datadriven approach, covariate relationships are tested for their statistical significance and based on both statistical significance and possible clinical relevance they are retained in a model. Advantages of a data-driven approach in a covariate analysis, is that the covariate-parameter relationship is supported by the data. However, because it is a descriptive, empirical method, the extrapolation potential may be debatable. Mechanistic covariate models potentially have better extrapolation 
potential as they are supported by scientific theories and in vitro and/or in vivo data (71). On the other hand, strong physiological and pharmacological evidence is yet needed for these approaches, confirmed by trial data and its PK(PD) model. Therefore, to date data-driven covariate analysis is considered the most achievable in most settings.

Beside categorical influence of the covariates, the population approach also allows for continuous covariate functions to quantify maturation profiles in parameters across the pediatric age range. In order to develop a maturation function for e.g. drug clearance from for example 1-18 years of age, pediatric patients across this age range should be included. In general, when these maturation functions have been derived, it is typically not advised to extrapolate these functions to younger individuals $<1$ year of age, including neonates, as extrapolations to neonates have almost never been successful (31). To make PK and/or PD predictions within this younger age range, these patients should also be included as these patients are not only difficult to predict, but they are also part of a very heterogeneous group with respect to body weight (almost tenfold difference) and age (0-30 days of age) (10).

In the pediatric population, body weight is often identified as the most statistical significant covariate on clearance and volume of distribution (65). When such a covariate-parameter relationship has been identified, one should realize that it describes the correlation between body weight and a PK parameter, which does not necessarily imply a causal relationship. Therefore caution is needed when extrapolating these models to subpopulations at the extremes of the age-adjusted body weight range, e.g. underfed or obese children. For example, Van Rongen et al. found that midazolam clearance in adolescent patients with overweight or obesity seemed not very different from literature values of adolescents with a healthy body mass index (72). While this implies that dosing in adolescents with obesity should not be expressed in $\mathrm{mg} / \mathrm{kg}$, in another example, i.e. busulfan, a weight-based algorithm proved to lead to predictable concentrations both in underweight and overweight individuals (73). Furthermore, even in pediatrics there are also special populations like patients with renal and/or hepatic impairment. Due to high variability in PK parameters during childhood and usually few available plasma samples per individual over a prolonged time interval, functional impairment may become apparent only after the growth spurt in adolescence, when significant decrease in GFR and/or increase in metabolic load can be measured (74). Therefore it can be hard to distinguish between functional impairment and maturation of clearance pathways in children. 


\section{Model validation}

When a trial is performed and a PKPD model has been developed, the model performance should be evaluated (75), which is a step that is often neglected (65). Marsot et al. have reviewed published population pharmacokinetic models developed in pediatric subjects from neonates to 2-year-old toddlers between 1985 and December 2010 (65). Disturbingly, the authors reported the use of model evaluation methods including bootstrap, cross-validation, Monte-Carlo simulations in only $37 \%$ of articles and the use of external validation methods in only $11 \%$ of articles. Without thorough model validation, predictions from simulations can however not be confirmed and therefore the level of certainty about the model-derived dosing recommendation is very low. Because it is essential to validate models if clinical decisions are being based on a model, there is obviously an urgent need for higher standards in the methodologies used to develop and publish pediatric pharmacological studies, with a need to systematically implement advanced model validation methods and external model validation.

Validation techniques include goodness-of-fit plots (76), and simulation-based diagnostics by means of for instance visual predictive checks $(\mathrm{VPC})(77,78)$ or normalized prediction distribution errors (NPDE) (79). Furthermore, bootstrapping methods can be used to evaluate the precision of model predictions and to calculate imprecision in model parameter estimates (80). These validation and/or evaluation tools are available, however they may need adaptations for use in the pediatric population (75). For example, goodness-of-fit plots should be stratified for different age or weight categories, to check for misspecification in covariate-parameter correlations $(75,76)$.

This internal validation should be followed by an external validation by applying the model to data from other studies, whenever available (75). This external validation is important to make sure that the model reflects the target population, instead of a model that only reflects the patients studied during model development. For example, morphine pharmacokinetics have been described by a PK model for morphine in children younger than 3 years of age (81) and this model has been externally evaluated with six other datasets from literature (82). Krekels et al. proved that the predictive performance of the morphine PK model was good in the external datasets of neonates and infants up to 1 year of age. It is important to consider which criteria are used in the model evaluation, as not all validation techniques allow to separate predictive models from models descriptive to one dataset only (75). The good predictive performance of a model is proven only once it has been both internally and externally validated. We emphasize that an external validation does not always require a new trial, as for commonly used drugs literature data may be available that can be used to evaluate the model's performance. For new drugs, it might be beneficial to split the data and use one third of the 
data to externally validate the model. This validated model can then be used for simulations to individualize drug regimens using a predefined therapeutic endpoint, e.g. an AUC/MIC < plasma concentration threshold or a PD endpoint like a sedation score.

\section{Prospective clinical trials for individualized dosing}

Validated models are not a goal in itself, but these models can be used to individualize drug regimen, using the covariates included in the model such as bodyweight, or gestational age (83). Before these dosing recommendations can be implemented in the clinic, a prospective clinical evaluation study should preferable be performed to ascertain that the desired endpoints are obtained using the novel dosing regimen (32). This prospective study should be seen as a first stage of implementation of the new dosing recommendations into clinical practice. So far in the period from 1985-2010, Marsot et al. found that only 48 out of 98 population PK studies concluded with dosing recommendations and none of these dosing recommendations were followed by a new study devoted to their clinical evaluation (65), which displays a need for tailoring of model-based dosing regimen, and their clinical evaluation. Wilbaux et al. describe in their review of pharmacometric models for primarily renally eliminated antibiotics in neonates, that despite many models and prospective trials for most of the reviewed antibiotics in children there is still no consensus on the optimal dosing regimen for most antibiotics (84).

However, an example of a prospective study in which an individualized dosing regimen is evaluated that was proposed based on a previously developed model, is amikacin dosing in preterm and term neonates. In this study, scenario-based simulations were performed using the developed PK model that identified both body weight and the postnatal age of neonates as covariates. This amikacin neonatal PK model (85) was used to derive a novel amikacin dosing regimen in neonates that was prospectively studied (86). The results of the prospective study show that the measured plasma concentrations were accurately predicted by the model and using the model-based dose recommendations, $90.5 \%$ and $60.2 \%$ of the patients had optimized peak and trough concentrations respectively (86). In this case, correcting for PK was sufficient, as success of treatment is measured by concentration, probably because target concentrations and exposure are bacteria specific and not age-specific.

In another example, Krekels et al. performed a study in which a model-based dosing regimen correcting for developmental difference in morphine PK (81), was prospectively evaluated in children receiving morphine after a surgery procedure (87). Their study revealed that the plasma 
concentrations were as expected based on the model predictions, and confirmed that the PK modelbased dosing regimen improved the exposure and led to clinical improvements. Moreover, it revealed that PK does not account for all inter-individual variability and that there is a need for investigation in PD variability to further optimize the dosing guideline as older children proved less sensitive to morphine than neonates (87).

The described model-based approach to guide dosing in children is mainly applied to small molecular drugs, but its value has also been proven for biologics. Thymoglobulin ${ }^{\circledR}$ is an anti-thymocyte globulin (ATG) that is used as serotherapy in patients undergoing hematopoietic cell transplantations (HTC). It is assumed that individualizing the ATG dosing to prevent graft-versus-host disease and rejection of transplants, will improve survival in pediatric HCT (88). Admiraal et al. described the pharmacokinetics of ATG in children and concluded after internal and external validation that the developed PK model was able to predict thymoglobulin concentrations in children correctly (88). Model-based simulations proved the current dosing regimens to be suboptimal leading to varying exposures between individuals, especially in patients with a baseline lymphocyte count that was lower than average and/or a body weight that was higher than average (88). To improve dosing, the relation between ATG exposure and clinical outcomes were studied and a PKPD model was developed to describe this relation (89). It was found that the exposure before transplantation of at least $40 \mathrm{AU} \times \mathrm{day} / \mathrm{mL}$ correlated with a decrease the incidence of graft-versus-host-disease and a lower incidence of graft failure (89). Using this model-based approach, quantification of these PKPD relation resulted in a dosing regimen to achieve the optimal exposure to improve efficacy and safety of ATG treatment in children undergoing transplantation (89), which is currently prospectively evaluated in clinical practice. 


\section{Expert commentary}

Over the past decades, the field of pharmacology has been evolving and modeling and simulation has started to play a role in improving pharmacotherapy in the pediatric population $(7,29)$. In general, more efforts should be put in the design of pediatric studies, so that the obtained data are the most informative to answer the research question. Furthermore, the data should always be analyzed with advanced statistical tools like population modeling to enable extraction of all this information and to perform proper covariate analyses. In addition, research efforts should focus on previously unstudied drugs, instead of performing new (PK) studies on drugs that have already been extensively studied in children, such as aminoglycosides in neonates on which many publications are yet available. For the latter, there would however still be much to gain in terms of translation of the results on the PK of these drugs into clinical practice (90) for instance by identifying subgroups for which these dosing guidelines may need further adaptation (e.g. neonates with asphyxia or renal insufficiency). However, before results of population models are used to guide dosing in clinical practice, it is imperative that these models are properly validated, both internally and externally, upon which the proposed model-based dosing regimen can be tested in a prospective trial. This next step, the translation of a model to novel dosing regimens, is often not taken (65), even though this is the most relevant step for improvement of pharmacotherapy in clinical care.

While more PK and PD information can be extracted from a trial on a specific drug in a specific age population when the population approach is used (90), it is not realistic to perform a trial and develop and validate a PK or PKPD model for each drug in each pediatric population. Therefore, further steps towards generating more generalizable, system-specific, information are also needed. In this respect, it has been hypothesized that pediatric PK models with covariate functions describing the ontogeny of drug clearance may contain drug-specific and physiological system-specific information(91). For example, the earlier mentioned neonatal model for GFR, originally developed to describe amikacin PK (10), was successfully used to predict ontogeny of clearance of other drugs eliminated by GFR $(85,92)$. However, more research on this type of extrapolation is needed, as it was reported that also drug properties may affect covariate relationships. For hepatically cleared drugs, it was for instance found that similarity in extraction ratio between the model drug and the drug the covariate relationship is extrapolated to, is important (93).

In addition, strategies should be developed to enable national formularies to adapt their dosing guidelines regularly based on the most recent advances in research published in literature, allowing clinicians to integrate guidelines in daily practice. For example, in gentamicin and tobramycin therapy in neonates, studies have shown that current dosing according to the Dutch National Formulary for 
Children (94), the British National Formulary for Children (95), Neofax (96) and the Red Book (97) leads to suboptimal exposure and inadequate target concentrations in neonates (98). Therefore, guidelines and national formularies should adapt to the most recent insights resulting in personalized medicine and improved clinical care. This is the case for gentamicin dosing in neonates, for which the Dutch National Formulary for Children (94) recently adjusted the dosing in premature neonates with a post-natal age of 0-7 days based on available literature.

\section{Five-year view}

In the next years, we anticipate a stronger trend towards the generation and identification of more generalizable information from pediatric clinical trials that have been performed on specific drugs. The trend has already been initiated by increased focus on research fields like PBPK modeling and systems pharmacology. Both fields integrate information from multiple clinical and/or preclinical sources to derive drug-specific and system-specific information. Identifying developmental patterns in system-specific parameters can help to quantify and predict inter-individual variability in drug absorption and disposition, and receptor function and expression (99). The advantage of systemsbased approaches in quantifying and predicting drug PK has for instance been illustrated for another special patient population. In morbidly obese and bariatric surgery patients, oral and intravenous midazolam PK was described by a semi-physiological PK model (100), taking into account both gut wall and liver processes. In this model, system-specific parameters (e.g. hepatic blood flow) and drug-specific parameters (e.g. extraction ratio) were combined, leading to the conclusion that in morbidly obese patients, hepatic CYP3A activity is reduced in comparison to healthy volunteers but normalizes one year after weight loss surgery (100). This approach does not require as much information as a full PBPK or systems pharmacology model, which is not always experimentally deducible. As some system-specific information is lacking, e.g. liver blood flow in special populations (100), different scenarios for this physiological parameter were tested for their influence on the conclusion. Ultimately, methods combining the population approach, based on outcome data, and the systems approach are being developed. In the near future we will see more of this approach in pediatric patients as well.

In order to describe the system and the maturation processes within the system, knowledge is required on disease mechanisms and drug action to be accounted for in the system models. Furthermore, to scale drug efficacy across a wide age range, the same endpoint to measure efficacy in children and adults is needed or scaling of endpoints between children and adults should be explored because PD endpoints used in adults cannot always be applied to children. For example, the 
gold standard for pain assessment in adults is based on self-report and in especially young children the same method cannot be applied. Therefore other validated and well-established scales like the COMFORT-B for pain after major surgery $(101,102)$ and PIPP pain scores for procedural pain in preterm neonates (103) have been used in preverbal children. It is important to validate these scores before use in clinical practice $(101,103)$, because even these pain scores give variable information about pain (104). The lack of validated PD endpoints in children is one of the most important obstacles in the transition of mostly PK studies to the more clinical relevant PKPD studies. Often it is assumed that a certain drug exposure that is associated with desired drug action in adults will produce the same response in children (105). However, this assumption does not hold true in many cases and therefore biomarkers for disease and drug efficacy in the pediatric populationare urgently needed to identify and quantify drug effects (105). To identify biomarkers for disease mechanisms and drug action in pediatrics, we foresee a trend in more interest in the metabolomics field (106, 107). Validated biomarkers and clear endpoints could also prevent pediatric trials to fail in answering the question addressed in the trial (108).

Predictive systems pharmacology models give novel insights in pediatric pharmacotherapy in relation to ontogeny and (patho)physiology, and advanced statistical techniques have been very instrumental in this field. As different types of data and multiple data analysis techniques are combined to integrate all information, pediatric pharmacological research will become even more multidisciplinary in nature. Not only in study design and data analysis, but also in implementation of novel dosing regimens in daily pharmacotherapy, professionals with different expertise are required to collaborate. There is an important urgency to actually use information that has been gathered on PK and/or PD of drugs in children into dosing recommendations through validated models and subsequent clinical trials. Ultimately all efforts should lead up to up-to-date formularies that can be used in clinical practice. To this end, approaches to accelerate the generation of dosing guidelines for drugs that have actually not yet been studied through PKPD modeling studies in children are needed for which physiologically based principles are promising. As such, in the next years, we anticipate a trend towards a systems-level approach in pediatric modeling to optimally use the information gained in pediatric trials. For this approach, properly designed clinical PKPD studies will remain the backbone of pediatric research. Ultimately, these efforts will lead to individualized drug treatment as standard pediatric care. 


\section{Key issues}

- In pediatric pharmacotherapy, many drugs are still used off-label, and their efficacy and safety is not well studied, even though developmental changes in children are known to lead to different pharmacokinetics (PK) and pharmacodynamics (PD) of drugs.

- Even though multiple PK(PD) studies have been performed particularly for many commonly used drugs, information obtained in these trials are not automatically translated into dosing regimens for clinical care.

- Proper study design can prevent structural and numerical identifiability of the PKPD model and clinical trial simulation and optimal design software provides a useful tool for optimizing the study design.

- As opportunities for plasma sampling in individual children are limited, the population approach is the preferred approach in PK and/or PD analyses to extract the greatest amount of PKPD information from the clinical data and perform covariate analyses.

- The optimal dosing regimen identified from a validated PKPD model should be preferably evaluated in a prospective clinical trial before implementation in clinical practice.

- Strategies should be developed to ensure that formularies update drug dosing guidelines regularly according to the most recent advances in research.

- More emphasis is needed to identify, quantify and validate biomarkers for disease and drug efficacy in the pediatric population.

- During the next few years, we anticipate a shift towards a systems-level approach in pediatric modeling in pediatric trials. For this approach, population PKPD studies will remain the backbone of pediatric research.

\section{Declaration of Interest}

The clinical research of K Allegaert is supported by the Fund for Scientific Research, Flanders (fundamental clinical investigatorship 1800214N) and the research activities are further facilitated by the agency for innovation by Science and Technology in Flanders (IWT) through the SAFEPEDRUG project (IWT/SBO 130033). CAJ Knibbe is supported by Innovational Research Incentives Scheme of the Dutch Organization for Scientific Research (NWO Vidi grant, May 2013 to CAJ Knibbe). The authors have no other relevant affiliations or financial involvement with any organization or entity with a financial interest in or financial conflict with the subject matter or materials discussed in the manuscript apart from those disclosed. 


\section{Reference annotations}

** Of considerable interest

- Admiraal, R., et al. (2014) Towards evidence-based dosing regimens in children on the basis of population pharmacokinetic pharmacodynamic modelling. Arch Dis Child 99(3), 267-72 Review on performing a population PKPD study and translating these results into evidencebased dosing regimens

- De Cock RF, Allegaert K, Sherwin CM, Nielsen El, de Hoog M, van den Anker JN, et al. A neonatal amikacin covariate model can be used to predict ontogeny of other drugs eliminated through glomerular filtration in neonates. Pharmaceutical research. 2014;31(3):754-67.

Systems approach: a neonatal GFR model can be used to predict PK of multiple drugs

- De Cock RF, Piana C, Krekels EH, Danhof M, Allegaert K, Knibbe CA, The role of population PKPD modelling in paediatric clinical research. European journal of clinical pharmacology. 2011;67 Suppl 1:5-16.

Overview on how modeling and simulation can help in pediatric clinical research

- Kearns GL, Artman M. Functional Biomarkers: an Approach to Bridge Pharmacokinetics and Pharmacodynamics in Pediatric Clinical Trials. Curr Pharm Des. 2015;21(39):5636-42.

Review on the need for biomarkers capable of measuring drug response in both a time and age dependent fashion

- Krekels EH, van Hasselt JG, Tibboel D, Danhof M, Knibbe CA. Systematic evaluation of the descriptive and predictive performance of paediatric morphine population models. Pharmaceutical research. 2011;28(4):797-811.

Overview of evaluation and validation techniques in children

Smits A, De Cock RF, Allegaert K, Vanhaesebrouck S, Danhof M, Knibbe CA. Prospective Evaluation of a Model-Based Dosing Regimen for Amikacin in Preterm and Term Neonates in Clinical Practice. Antimicrob Agents Chemother. 2015;59(10):6344-51.

Prime example of a prospective trial to evaluate a model-based dosing regimen

- Valitalo PA, van den Anker JN, Allegaert K, de Cock RF, de Hoog M, Simons SH, et al. Novel model-based dosing guidelines for gentamicin and tobramycin in preterm and term 
neonates. J Antimicrob Chemother. 2015;70(7):2074-7.

Prime example of an evidence-based dosing regimen proposed after model development and validation

- Zhao W, Biran V, Jacqz-Aigrain E. Amikacin maturation model as a marker of renal maturation to predict glomerular filtration rate and vancomycin clearance in neonates. Clin Pharmacokinet. 2013;52(12):1127-34.

External validation where a neonatal GFR model is used to predict vancomycin PK

* Of interest

- Bellanti F, Della Pasqua O. Modelling and simulation as research tools in paediatric drug development. Eur J Clin Pharmacol. 2011;67 Suppl 1:75-86.

Literature review that concludes that a consensus is lacking in how to assess the impact of developmental factors on efficacy and safety of pharmacotherapy in children

- Joseph PD, Craig JC, Caldwell PH. Clinical trials in children. Br J Clin Pharmacol. 2015;79(3):357-69.

Recent review of challenges and solutions for performing clinical trials in children

- Krekels EH, Tibboel D, de Wildt SN, Ceelie I, Dahan A, van Dijk M, et al. Evidence-based morphine dosing for postoperative neonates and infants. Clin Pharmacokinet. 2014;53(6):553-63.

Prime example of a prospective trial to evaluate a model-based dosing regimen

- Krekels EH, DeJongh J, van Lingen RA, van der Marel CD, Choonara I, Lynn AM, et al. Predictive performance of a recently developed population pharmacokinetic model for morphine and its metabolites in new datasets of (preterm) neonates, infants and children. Clin Pharmacokinet. 2011;50(1):51-63.

Prime example of an external model validation

- $\quad$ Lu H, Rosenbaum S. Developmental pharmacokinetics in pediatric populations. J Pediatr Pharmacol Ther. 2014;19(4):262-76.

Physiological changes affecting pharmacokinetics in children 
- Marsot A, Boulamery A, Bruguerolle B, Simon N. Population pharmacokinetic analysis during the first 2 years of life: an overview. Clin Pharmacokinet. 2012;51(12):787-98.

Overview of PK analysis with proper validation, prospective trials and development of dosing regimens

- Thomson AH, Elliott HL. Designing simple PK-PD studies in children. Paediatr Anaesth. 2011;21(3):190-6.

Review on how to design efficacy trials in children for population PKPD analysis

- van Dijk M, Ceelie I, Tibboel D. Endpoints in pediatric pain studies. Eur J Clin Pharmacol. 2011;67 Suppl 1:61-6.

Clear illustration of the lack of pharmacodynamic data in pain studies in children

\section{References}

1. Balan S, Hassali MA, Mak VS. Awareness, knowledge and views of off-label prescribing in children: a systematic review. Br J Clin Pharmacol. 2015;80(6):1269-80.

2. Joseph PD, Craig JC, Caldwell PH. Clinical trials in children. Br J Clin Pharmacol. 2015;79(3):357-69.

3. Shaddy RE, Denne SC, Committee on D, Committee on Pediatric R. Clinical report--guidelines for the ethical conduct of studies to evaluate drugs in pediatric populations. Pediatrics. 2010;125(4):850-60.

4. Rosato J. The ethics of clinical trials: a child's view. J Law Med Ethics. 2000;28(4):362-78.

5. van Dijk M, Ceelie I, Tibboel D. Endpoints in pediatric pain studies. Eur J Clin Pharmacol. 2011:67 Suppl 1:61-6.

6. Mahmood 1. Dosing in children: a critical review of the pharmacokinetic allometric scaling and modelling approaches in paediatric drug development and clinical settings. Clin Pharmacokinet. 2014;53(4):327-46.

7. Wang J, Avant D, Green D, Seo S, Fisher J, Mulberg AE, et al. A Survey of Neonatal Pharmacokinetic and Pharmacodynamic Studies in Pediatric Drug Development. Clin Pharmacol Ther. 2015;98(3):328-35.

8. Johnson TN, Rostami-Hodjegan A. Resurgence in the use of physiologically based pharmacokinetic models in pediatric clinical pharmacology: parallel shift in incorporating the knowledge of biological elements and increased applicability to drug development and clinical practice. Paediatr Anaesth. 2011;21(3):291-301. 
9. Edginton AN, Schmitt W, Voith B, Willmann S. A mechanistic approach for the scaling of clearance in children. Clin Pharmacokinet. 2006;45(7):683-704.

10. De Cock RF, Allegaert K, Schreuder MF, Sherwin CM, de Hoog M, van den Anker JN, et al. Maturation of the glomerular filtration rate in neonates, as reflected by amikacin clearance. Clin Pharmacokinet. 2012;51(2):105-17.

11. Anderson BJ, Holford NH. Mechanistic basis of using body size and maturation to predict clearance in humans. Drug Metab Pharmacokinet. 2009;24(1):25-36.

12. Strougo A, Eissing T, Yassen A, Willmann S, Danhof M, Freijer J. First dose in children: physiological insights into pharmacokinetic scaling approaches and their implications in paediatric drug development. J Pharmacokinet Pharmacodyn. 2012;39(2):195-203.

13. Johnson TN. The problems in scaling adult drug doses to children. Arch Dis Child. 2008;93(3):207-11.

14. Janiaud P, Lajoinie A, Cour-Andlauer F, Cornu C, Cochat P, Cucherat M, et al. Different treatment benefits were estimated by clinical trials performed in adults compared with those performed in children. J Clin Epidemiol. 2015;68(10):1221-31.

15. Lu H, Rosenbaum S. Developmental pharmacokinetics in pediatric populations. J Pediatr Pharmacol Ther. 2014;19(4):262-76.

16. Johnson TN. The development of drug metabolising enzymes and their influence on the susceptibility to adverse drug reactions in children. Toxicology. 2003;192(1):37-48.

17. Kearns GL, Abdel-Rahman SM, Alander SW, Blowey DL, Leeder JS, Kauffman RE. Developmental pharmacology--drug disposition, action, and therapy in infants and children. N Engl J Med. 2003;349(12):1157-67.

18. Bjorkman S. Prediction of drug disposition in infants and children by means of physiologically based pharmacokinetic (PBPK) modelling: theophylline and midazolam as model drugs. Br J Clin Pharmacol. 2005;59(6):691-704.

19. Alcorn J, McNamara PJ. Ontogeny of hepatic and renal systemic clearance pathways in infants: part II. Clin Pharmacokinet. 2002;41(13):1077-94.

20. Hines RN. Developmental expression of drug metabolizing enzymes: impact on disposition in neonates and young children. Int J Pharm. 2013;452(1-2):3-7.

21. de Wildt SN, Kearns GL, Leeder JS, van den Anker JN. Cytochrome P450 3A: ontogeny and drug disposition. Clin Pharmacokinet. 1999;37(6):485-505.

22. Pang KS, Rowland M. Hepatic clearance of drugs. I. Theoretical considerations of a "wellstirred" model and a "parallel tube" model. Influence of hepatic blood flow, plasma and blood cell binding, and the hepatocellular enzymatic activity on hepatic drug clearance. $J$ Pharmacokinet Biopharm. 1977;5(6):625-53. 
23. Rhodin MM, Anderson BJ, Peters AM, Coulthard MG, Wilkins B, Cole M, et al. Human renal function maturation: a quantitative description using weight and postmenstrual age. Pediatr Nephrol. 2009;24(1):67-76.

24. Mahmood I, Staschen CM. Prediction of Human Glomerular Filtration Rate from Preterm Neonates to Adults: Evaluation of Predictive Performance of Several Empirical Models. AAPS J. 2016;18(2):445-54.

25. Sethi PK, White CA, Cummings BS, Hines RN, Muralidhara S, Bruckner JV. Ontogeny of plasma proteins, albumin and binding of diazepam, cyclosporine, and deltamethrin. Pediatr Res. 2016;79(3):409-15.

26. McNamara PJ, Alcorn J. Protein binding predictions in infants. AAPS PharmSci. 2002;4(1):E4.

27. Grand RJ, Watkins JB, Torti FM. Development of the human gastrointestinal tract. A review. Gastroenterology. 1976;70(5 PT.1):790-810.

28. Van Den Anker JN VU-DA, Woestenborghs R, Koster M and Sauer PJJ. The effect of gastric pH on the absorption of ketoconazole by very-low-birth-weight infants. Pediatric Research. 1994;36:4A-A.

29. Bellanti F, Della Pasqua O. Modelling and simulation as research tools in paediatric drug development. Eur J Clin Pharmacol. 2011;67 Suppl 1:75-86.

30. Abdel-Rahman SM, Amidon GL, Kaul A, Lukacova V, Vinks AA, Knipp GT, et al. Summary of the National Institute of Child Health and Human Development-best pharmaceuticals for Children Act Pediatric Formulation Initiatives Workshop-Pediatric Biopharmaceutics Classification System Working Group. Clin Ther. 2012;34(11):S11-24.

31. Cella M, Zhao W, Jacqz-Aigrain E, Burger D, Danhof M, Della Pasqua O. Paediatric drug development. are population models predictive of pharmacokinetics across paediatric populations? Br J Clin Pharmacol. 2011;72(3):454-64.

32. Incel, de Wildt SN, Tibboel D, Danhof M, Knibbe CA. Tailor-made drug treatment for children: creation of an infrastructure for data-sharing and population PK-PD modeling. Drug Discov Today. 2009;14(5-6):316-20.

33. Sheiner LB. Learning versus confirming in clinical drug development. Clin Pharmacol Ther. 1997;61(3):275-91.

34. Ette EI, Williams PJ, Lane JR. Population pharmacokinetics III: design, analysis, and application of population pharmacokinetic Studies. Ann Pharmacother. 2004;38(12):2136-44.

35. Beal SL, Sheiner LB. Estimating population kinetics. Crit Rev Biomed Eng. 1982;8(3):195-222.

36. Sheiner LB, Beal SL. Evaluation of methods for estimating population pharmacokinetics parameters. I. Michaelis-Menten model: routine clinical pharmacokinetic data. J

Pharmacokinet Biopharm. 1980;8(6):553-71. 
37. Wade JR, Beal SL, Sambol NC. Interaction between structural, statistical, and covariate models in population pharmacokinetic analysis. J Pharmacokinet Biopharm. 1994;22(2):16577.

38. De Cock RF, Piana C, Krekels EH, Danhof M, Allegaert K, Knibbe CA. The role of population PKPD modelling in paediatric clinical research. European journal of clinical pharmacology. 2011;67 Suppl 1:5-16.

39. Jonsson EN, Wade JR, Karlsson MO. Nonlinearity detection: advantages of nonlinear mixedeffects modeling. AAPS PharmSci. 2000;2(3):E32.

40. Thomson AH, Elliott HL. Designing simple PK-PD studies in children. Paediatr Anaesth. 2011;21(3):190-6.

41. Bartelink IH, Rademaker CM, Schobben AF, van den Anker JN. Guidelines on paediatric dosing on the basis of developmental physiology and pharmacokinetic considerations. Clin Pharmacokinet. 2006;45(11):1077-97.

42. EMA. EMA (EMA/CHMP/EWP/147013/2004 Committee for Medicinal Products for Human Use [CHMP]). Guideline on the role of pharmacokinetics in the development of medicinal products in the paediatric population. 2007.

43. EMA. EMA (EMA/CHMP/QWP/805880/2012 Rev.2. Committe for medicinal Products for Human Use [CHMP] Paediatric Committee [PDCO]). Guideline on pharmaceutical development of medicines for paediatric use. 2014.

44. FDA. (U.S. Department of Health and Human Services Food and Drug Administration Center for Drug Evaluation and Research (CDER) Clinical Pharmacology) December 2014, General Clinical Pharmacology Considerations for Pediatric Studies for Drugs and Biological Products Guidance for Industry. . 2014.

45. Goodman SN, Sladky JT. A Bayesian approach to randomized controlled trials in children utilizing information from adults: the case of Guillain-Barre syndrome. Clin Trials. 2005;2(4):305-10; discussion 64-78.

46. Milton MN, Horvath CJ. The EMEA guideline on first-in-human clinical trials and its impact on pharmaceutical development. Toxicol Pathol. 2009;37(3):363-71.

47. Manolis E, Pons G. Proposals for model-based paediatric medicinal development within the current European Union regulatory framework. Br J Clin Pharmacol. 2009;68(4):493-501.

48. Meibohm B, Laer S, Panetta JC, Barrett JS. Population pharmacokinetic studies in pediatrics: issues in design and analysis. AAPS J. 2005;7(2):E475-87.

49. Barker $\mathrm{Cl}$, Germovsek E, Hoare RL, Lestner JM, Lewis J, Standing JF. Pharmacokinetic/pharmacodynamic modelling approaches in paediatric infectious diseases and immunology. Adv Drug Deliv Rev. 2014;73:127-39. 
50. Jacqmin P, Snoeck E, van Schaick EA, Gieschke R, Pillai P, Steimer JL, et al. Modelling response time profiles in the absence of drug concentrations: definition and performance evaluation of the K-PD model. J Pharmacokinet Pharmacodyn. 2007;34(1):57-85.

51. Barrett JS, Della Casa Alberighi O, Laer S, Meibohm B. Physiologically based pharmacokinetic (PBPK) modeling in children. Clin Pharmacol Ther. 2012;92(1):40-9.

52. Tayman C, Rayyan M, Allegaert K. Neonatal pharmacology: extensive interindividual variability despite limited size. J Pediatr Pharmacol Ther. 2011;16(3):170-84.

53. Evans ND, Godfrey KR, Chapman MJ, Chappell MJ, Aarons L, Duffull SB. An identifiability analysis of a parent-metabolite pharmacokinetic model for ivabradine. J Pharmacokinet Pharmacodyn. 2001;28(1):93-105.

54. Ahlers SJ, Valitalo PA, Peeters MY, Gulik L, van Dongen EP, Dahan A, et al. Morphine Glucuronidation and Elimination in Intensive Care Patients: A Comparison with Healthy Volunteers. Anesth Analg. 2015;121(5):1261-73.

55. Shivva V, Korell J, Tucker IG, Duffull SB. An approach for identifiability of population pharmacokinetic-pharmacodynamic models. CPT Pharmacometrics Syst Pharmacol. 2013;2:e49.

56. Sheiner LB, Beal SL. Evaluation of methods for estimating population pharmacokinetic parameters. III. Monoexponential model: routine clinical pharmacokinetic data. J Pharmacokinet Biopharm. 1983;11(3):303-19.

57. Hashimoto $Y$, Sheiner LB. Designs for population pharmacodynamics: value of pharmacokinetic data and population analysis. J Pharmacokinet Biopharm. 1991;19(3):33353.

58. al-Banna MK, Kelman AW, Whiting B. Experimental design and efficient parameter estimation in population pharmacokinetics. J Pharmacokinet Biopharm. 1990;18(4):347-60.

59. Ette El, Howie CA, Kelman AW, Whiting B. Experimental design and efficient parameter estimation in preclinical pharmacokinetic studies. Pharm Res. 1995;12(5):729-37.

60. Aarons L, Balant LP, Mentre F, Morselli PL, Rowland M, Steimer JL, et al. Practical experience and issues in designing and performing population pharmacokinetic/pharmacodynamic studies. Eur J Clin Pharmacol. 1996;49(4):251-4.

61. Ogungbenro K, Matthews I, Looby M, Kaiser G, Graham G, Aarons L. Population pharmacokinetics and optimal design of paediatric studies for famciclovir. Br J Clin Pharmacol. 2009;68(4):546-60.

62. Nyberg J, Bazzoli C, Ogungbenro K, Aliev A, Leonov S, Duffull S, et al. Methods and software tools for design evaluation in population pharmacokinetics-pharmacodynamics studies. $\mathrm{Br} \mathrm{J}$ Clin Pharmacol. 2015;79(1):6-17. 
63. Bouillon-Pichault M, Jullien V, Bazzoli C, Pons G, Tod M. Pharmacokinetic design optimization in children and estimation of maturation parameters: example of cytochrome P450 3A4. J Pharmacokinet Pharmacodyn. 2011;38(1):25-40.

64. Roberts JK, Stockmann C, Balch A, Yu T, Ward RM, Spigarelli MG, et al. Optimal design in pediatric pharmacokinetic and pharmacodynamic clinical studies. Paediatr Anaesth. 2015;25(3):222-30.

65. Marsot A, Boulamery A, Bruguerolle B, Simon N. Population pharmacokinetic analysis during the first 2 years of life: an overview. Clin Pharmacokinet. 2012;51(12):787-98.

66. Wildschut ED, de Wildt SN, Mathot RA, Reiss IK, Tibboel D, Van den Anker J. Effect of hypothermia and extracorporeal life support on drug disposition in neonates. Semin Fetal Neonatal Med. 2013;18(1):23-7.

67. Wildschut ED, Ahsman MJ, Houmes RJ, Pokorna P, de Wildt SN, Mathot RA, et al. Pharmacotherapy in neonatal and pediatric extracorporeal membrane oxygenation (ECMO). Curr Drug Metab. 2012;13(6):767-77.

68. Bonate PL. The effect of collinearity on parameter estimates in nonlinear mixed effect models. Pharm Res. 1999;16(5):709-17.

69. Bolton SaB, C. "Choosing samples," in Pharmaceutical Statistics Practical and Clinical Applications, Fifth edit., New York: Informa Healthcare USA, 2010, p. 76. 2010.

70. van Hasselt JG, Schellens JH, Beijnen JH, Huitema AD. Design of informative renal impairment studies: evaluation of the impact of design stratification on bias, precision and dose adjustment error. Invest New Drugs. 2014;32(5):913-27.

71. Jamei M, Dickinson GL, Rostami-Hodjegan A. A framework for assessing inter-individual variability in pharmacokinetics using virtual human populations and integrating general knowledge of physical chemistry, biology, anatomy, physiology and genetics: A tale of 'bottom-up vs 'top-down' recognition of covariates. Drug Metab Pharmacokinet. 2009,24(1):53-75.

72. van Rongen A, Vaughns JD, Moorthy GS, Barrett JS, Knibbe CA, van den Anker JN. Population pharmacokinetics of midazolam and its metabolites in overweight and obese adolescents. $\mathrm{Br}$ J Clin Pharmacol. 2015;80(5):1185-96.

73. Bartelink IH, van Kesteren C, Boelens JJ, Egberts TC, Bierings MB, Cuvelier GD, et al. Predictive performance of a busulfan pharmacokinetic model in children and young adults. Ther Drug Monit. 2012;34(5):574-83.

74. Goldstein SL. Renal recovery at different ages. Nephron Clin Pract. 2014;127(1-4):21-4. 
75. Krekels EH, van Hasselt JG, Tibboel D, Danhof M, Knibbe CA. Systematic evaluation of the descriptive and predictive performance of paediatric morphine population models. Pharmaceutical research. 2011;28(4):797-811.

76. Karlsson MO, Savic RM. Diagnosing model diagnostics. Clin Pharmacol Ther. 2007;82(1):1720.

77. Karlsson M, Holford, N. A tutorial on visual predictive checks. PAGE 17 (2008)2008. p. Abstr 1434 [www.page-meeting.org/?abstract=].

78. Holford N. The visual predictive check-superiority to standard diagnostic (Rorschach) plots. PAGE 14 (2005)2005. p. Abstr 738 [www.page-meeting.org/?abstract=].

79. Brendel K, Comets E, Laffont C, Laveille C, Mentre F. Metrics for external model evaluation with an application to the population pharmacokinetics of gliclazide. Pharm Res. 2006;23(9):2036-49.

80. Lindbom L, Pihlgren P, Jonsson EN. PsN-Toolkit--a collection of computer intensive statistical methods for non-linear mixed effect modeling using NONMEM. Comput Methods Programs Biomed. 2005;79(3):241-57.

81. Knibbe CA, Krekels EH, van den Anker JN, DeJongh J, Santen GW, van Dijk M, et al. Morphine glucuronidation in preterm neonates, infants and children younger than 3 years. Clin Pharmacokinet. 2009;48(6):371-85.

82. Krekels EH, DeJongh J, van Lingen RA, van der Marel CD, Choonara I, Lynn AM, et al. Predictive performance of a recently developed population pharmacokinetic model for morphine and its metabolites in new datasets of (preterm) neonates, infants and children. Clin Pharmacokinet. 2011,50(1):51-63.

83. Zhao W, Zhang D, Storme T, Baruchel A, Decleves X, Jacqz-Aigrain E. Population pharmacokinetics and dosing optimization of teicoplanin in children with malignant haematological disease. Br J Clin Pharmacol. 2015;80(5):1197-207.

84. Wilbaux M, Fuchs A, Samardzic J, Rodieux F, Csajka C, Allegaert K, et al. Pharmacometric Approaches to Personalize Use of Primarily Renally Eliminated Antibiotics in Preterm and Term Neonates. J Clin Pharmacol. 2016.

85. De Cock RF, Allegaert K, Sherwin CM, Nielsen El, de Hoog M, van den Anker JN, et al. A neonatal amikacin covariate model can be used to predict ontogeny of other drugs eliminated through glomerular filtration in neonates. Pharmaceutical research. 2014;31(3):754-67.

86. Smits A, De Cock RF, Allegaert K, Vanhaesebrouck S, Danhof M, Knibbe CA. Prospective Evaluation of a Model-Based Dosing Regimen for Amikacin in Preterm and Term Neonates in Clinical Practice. Antimicrob Agents Chemother. 2015;59(10):6344-51. 
87. Krekels EH, Tibboel D, de Wildt SN, Ceelie I, Dahan A, van Dijk M, et al. Evidence-based morphine dosing for postoperative neonates and infants. Clin Pharmacokinet.

2014;53(6):553-63.

88. Admiraal R, van Kesteren C, Jol-van der Zijde CM, van Tol MJ, Bartelink IH, Bredius RG, et al. Population pharmacokinetic modeling of Thymoglobulin $((\mathrm{R}))$ in children receiving allogeneichematopoietic cell transplantation: towards improved survival through individualized dosing. Clin Pharmacokinet. 2015;54(4):435-46.

89. Admiraal R, van Kesteren C, Jol-van der Zijde CM, Lankester AC, Bierings MB, Egberts TC, et al. Association between anti-thymocyte globulin exposure and CD4+ immune reconstitution in paediatric haemopoietic cell transplantation: a multicentre, retrospective pharmacodynamic cohort analysis. Lancet Haematol. 2015;2(5):e194-203.

90. Admiraal R, van Kesteren C, Boelens JJ, Bredius RG, Tibboel D, Knibbe CA. Towards evidencebased dosing regimens in children on the basis of population pharmacokinetic pharmacodynamic modelling. Arch Dis Child. 2014;99(3):267-72.

91. Krekels EH, Tibboel D, Knibbe CA. Pediatric pharmacology: current efforts and future goals to improve clinical practice. Expert Opin Drug Metab Toxicol. 2015;11(11):1679-82.

92. Zhao W, Biran V, Jacqz-Aigrain E. Amikacin maturation model as a marker of renal maturation to predict glomerular filtration rate and vancomycin clearance in neonates. Clin Pharmacokinet. 2013;52(12):1127-34.

93. Calvier EAM KE, Knibbe CAJ. Extrapolation potential of semi-physiological covariate models to newborns: a simulation-based study. PAGE 24 (2015) Abstr 3595 [www.pagemeeting.org/?abstract=3595]. 2015.

94. Kinderformularium. Dutch Knowledge Centre for Pharmacotherapy in Children. Dutch National Formulary for Children/Kinderformularium. http://www.kinderformularium.nl/.

95. BNFC. Paediatric Formulary Committee. British National Formulary for Children. London: BMJ Group, 2009.

96. Neofax. Young TE. Neofax. Montvale: Thomson Reuters, 2011.

97. Book R. Pickering LK, Baker CJ, Kimberlin DW. Red Book: 2012 Report of the Committee on Infectious Diseases. American Academy of Pediatrics, 2012.

98. Valitalo PA, van den Anker JN, Allegaert K, de Cock RF, de Hoog M, Simons SH, et al. Novel model-based dosing guidelines for gentamicin and tobramycin in preterm and term neonates. J Antimicrob Chemother. 2015;70(7):2074-7.

99. van der Graaf PH, Benson N. Systems pharmacology: bridging systems biology and pharmacokinetics-pharmacodynamics (PKPD) in drug discovery and development. Pharm Res. 2011;28(7):1460-4. 
100. Brill MJ, Valitalo PA, Darwich AS, van Ramshorst B, van Dongen HP, Rostami-Hodjegan A, et al. Semiphysiologically based pharmacokinetic model for midazolam and CYP3A mediated metabolite 1-OH-midazolam in morbidly obese and weight loss surgery patients. CPT Pharmacometrics Syst Pharmacol. 2016;5(1):20-30.

101. Ista $E$, van Dijk M, Tibboel D, de Hoog M. Assessment of sedation levels in pediatric intensive care patients can be improved by using the COMFORT "behavior" scale. Pediatr Crit Care Med. 2005;6(1):58-63.

102. Carnevale FA, Razack S. An item analysis of the COMFORT scale in a pediatric intensive care unit. Pediatr Crit Care Med. 2002;3(2):177-80.

103. Stevens B, Johnston C, Petryshen P, Taddio A. Premature Infant Pain Profile: development and initial validation. Clin J Pain. 1996;12(1):13-22.

104. Valitalo PA, van Dijk M, Krekels EH, Gibbins S, Simons SH, Tibboel D, et al. Pain and distress caused by endotracheal suctioning in neonates is better quantified by behavioural than physiological items: A comparison based on Item Response Theory modelling. Pain. 2016.

105. Kearns GL, Artman M. Functional Biomarkers: an Approach to Bridge Pharmacokinetics and Pharmacodynamics in Pediatric Clinical Trials. Curr Pharm Des. 2015;21(39):5636-42.

106. Mussap M, Noto A, Cibecchini F, Fanos V. The importance of biomarkers in neonatology. Semin Fetal Neonatal Med. 2013;18(1):56-64.

107. Fanos V, Barberini L, Antonucci R, Atzori L. Metabolomics in neonatology and pediatrics. Clin Biochem. 2011;44(7):452-4.

108. Momper JD, Mulugeta Y, Burckart GJ. Failed Pediatric Drug Development Trials. Clin Pharmacol Ther. 2015;98(3):245-51. 